MATHEMATICS OF COMPUTATION

Volume 66, Number 217, January 1997, Pages 69-84

S 0025-5718(97)00804-1

\title{
DECAY RATE FOR PERTURBATIONS OF STATIONARY DISCRETE SHOCKS FOR CONVEX SCALAR CONSERVATION LAWS
}

\author{
HAILIANG LIU AND JINGHUA WANG
}

\begin{abstract}
This paper is to study the decay rate for perturbations of stationary discrete shocks for the Lax-Friedrichs scheme approximating the scalar conservation laws by means of an elementary weighted energy method. If the summation of the initial perturbation over $(-\infty, j)$ is small and decays at the algebraic rate as $|j| \rightarrow \infty$, then the solution approaches the stationary discrete shock profiles at the corresponding rate as $n \rightarrow \infty$. This rate seems to be almost optimal compared with the rate in the continuous counterpart. Proofs are given by applying the weighted energy integration method to the integrated scheme of the original one. The selection of the time-dependent discrete weight function plays a crucial role in this procedure.
\end{abstract}

\section{INTRODUCTION}

In this paper, we continue to study the asymptotic stability of the Lax-Friedrichs (LF) scheme

$$
u_{j}^{n+1}-u_{j}^{n}+\frac{\lambda}{2}\left(f\left(u_{j+1}^{n}\right)-f\left(u_{j-1}^{n}\right)\right)=\frac{\mu}{2}\left(u_{j+1}^{n}-2 u_{j}^{n}+u_{j-1}^{n}\right)
$$

approximating the convex scalar conservation laws

$$
u_{t}+f(u)_{x}=0, \quad u(x, 0)=u_{0}(x)= \begin{cases}u_{-}, & x<0, \\ u_{+}, & x>0 .\end{cases}
$$

The corresponding shock wave solution is

$$
u(t, x)= \begin{cases}u_{-}, & x-s t<0 \\ u_{+}, & x-s t>0\end{cases}
$$

where the end states $u_{ \pm}$related shock speed $s$ by the Rankine-Hugoniot condition

$$
-s\left(u_{+}-u_{-}\right)+f\left(u_{+}\right)-f\left(u_{-}\right)=0,
$$

and Oleinik's shock condition

$$
\left(u_{+}-u_{-}\right) Q \equiv\left(u_{+}-u_{-}\right)\left[f(u)-f\left(u_{ \pm}\right)-s\left(u-u_{ \pm}\right)\right]>0
$$

Received by the editor May 19, 1995 and, in revised form, January 31, 1996.

1991 Mathematics Subject Classification. Primary 39A11; Secondary 35L65.

Key words and phrases. Decay rate, discrete shocks, Lax-Friedrichs scheme.

The first author was supported in part by the National Natural Science Foundation of China and by the Institute of Mathematics, Academia Sinica.

The second author was supported in part by the National Natural Science Foundation of China and by The Texs Coordinating Board for Higher Education, Advanced Research Program.

(C)1997 American Mathematical Society 
for $u \in\left(\min \left(u_{-}, u_{+}\right), \max \left(u_{-}, u_{+}\right)\right)$. It is noted that when $s \neq f^{\prime}\left(u_{ \pm}\right)(1.3 \mathrm{~b})$ implies the Lax shock condition

$$
f^{\prime}\left(u_{+}\right)<s<f^{\prime}\left(u_{-}\right)
$$

Let $x_{j}=j r$ and $t_{n}=n h$, with $r=\Delta x$ and $h=\Delta t$ being the spatial and the temporal grid sizes. Denote an approximation of $u\left(x_{j}, t_{n}\right)$ by $u_{j}^{n}, \mu$ is a constant satisfying $0<\mu<1$, and the temporal and spatial grid ratio $\lambda=\frac{\Delta t}{\Delta x}$ satisfies the Courant-Friedrichs-Levy (CFL) condition,

$$
\lambda \max \left|f^{\prime}\right| \leq \mu .
$$

Corresponding to the difference equation (1.1) we have the following viscous conservation law

$$
u_{t}+f(u)_{x}=\epsilon u_{x x}, \epsilon>0,
$$

which has a viscous shock profile solution $u=U(x-s t)$ satisfying

$$
U(z) \rightarrow u_{ \pm} \text {as } z \rightarrow \pm \infty .
$$

For convenience, we assume that $u_{+}<u_{-}$and $s \neq f^{\prime}\left(u_{ \pm}\right)$, then $U^{\prime}(z)<0$ and $\left|U-u_{ \pm}\right| \sim O(1) \exp (-c|z|)$ as $z \rightarrow \pm \infty$. Hence the shock profile of (1.5) has the following property

$$
u(x, t+\Delta t)=u(x-s \Delta t, t) .
$$

Since the solutions of the difference equation are only defined on the grid nodes, (1.6) does not always make sense. For simplicity, we focus on the stationary discrete shock profile solution $\phi_{j}$ of (1.1) i.e.,

$$
\begin{gathered}
\lambda\left(f\left(\phi_{j+1}\right)-f\left(\phi_{j-1}\right)\right)=\mu\left(\phi_{j+1}-2 \phi_{j}+\phi_{j-1}\right), \\
\phi_{j} \rightarrow u_{ \pm} \text {as } j \rightarrow \pm \infty .
\end{gathered}
$$

Its existence and properties have been proved by Jennings [7] provided that $\left(u_{-}, u_{+}\right)$ satisfies (1.3a)-(1.3b).

Consider the initial value problem for (1.1) with the initial condition

$$
u_{j}^{0} \rightarrow u_{ \pm} \quad \text { as } j \rightarrow \pm \infty
$$

and

$$
\sum_{j}\left(1+j^{2}\right)^{3 / 2}\left|u_{j}^{0}-\phi_{j}\right|^{2} \leq c_{1}
$$

for some (suitably small) positive constant $c_{1}$. Under these assumptions and $f$ being non-convex Liu and Wang [13] successfully proved that as $n \rightarrow \infty$, the solution $u_{j}^{n}$ of (1.1), (1.8) tends uniformly with respect to $j \in Z$, in maximum norm, to $\phi_{j}$ which is uniquely determined by the relation

$$
\sum_{j}\left(u_{j}^{0}-\phi_{j}\right)=0
$$

In [13], we proved that the above discrete shocks for the L-F scheme (1.1) is asymptotically stable in the $l^{2}$ - and $l^{\infty}$-norm. But, as far as we know, the decay rate is not known even in the case when $f$ is convex. For this reason, the asymptotic stability theory of discrete shock is not complete as yet. The main contribution 
of this article is to show the decay rate for a convex flux $f$ under some additional assumptions on initial data. We assume that the summation

$$
v_{j}^{0}=\sum_{-\infty}^{j}\left(u_{j}^{0}-\phi_{j}\right) \in l_{\alpha}^{2}
$$

exists for any $j \in Z$ and denote $\left|v_{j}^{0}\right|_{\alpha}=\left[\sum_{j}\left(1+(j r)^{2}\right)^{\alpha}\left(v_{j}^{0}\right)^{2}\right]^{1 / 2}$.

Then our main theorem is given as follows.

Theorem 1.1. Suppose (1.3a)-(1.3c) and (1.4) (CFL condition) hold. Let $\phi_{j}$ be a stationary discrete shock profile defined by (1.7a) connecting $u_{+}$to $u_{-}$. Suppose the initial data $u_{j}^{0}$ satisfy (1.10) and, for some $\alpha>0$,

$$
\sum_{j=-\infty}^{\infty}\left(1+j^{2}\right)^{\kappa}\left|u_{j}^{0}-\phi_{j}\right|^{2} \leq \delta_{1} \quad\left(\kappa>\frac{\alpha}{2}+1\right)
$$

for some (suitably small) positive constant $\delta_{1}$, then the unique global solution $u_{j}^{n}$, to the $L-F$ scheme (1.1) with initial data $u_{j}^{0}$ tends in the maximum norm to the shock profile $\phi_{j}$ at the following rate: If $\alpha$ is an integer, then it holds

$$
\sup _{j}\left|u_{j}^{n}-\phi_{j}\right| \leq C(1+n h)^{-\alpha / 2}\left|v_{j}^{0}\right|_{\alpha}, \quad n \geq 0,
$$

while if $\alpha$ is not an integer, then

$$
\sup _{j}\left|u_{j}^{n}-\phi_{j}\right| \leq C_{\varepsilon}(1+n h)^{-\mu / 2+\varepsilon}\left|v_{j}^{0}\right|_{\alpha}, \quad n \geq 0,
$$

for any constant $\varepsilon>0$ and some constant $C_{\varepsilon}$ such that $C_{\varepsilon} \rightarrow \infty$ as $\varepsilon \rightarrow 0$.

The study of existence and stability of discrete shocks is important in understanding the convergence behavior of numerical shock computations. Jennings [7] first investigated the existence and stability of discrete shocks for scalar difference equations. But the work is only restricted to the strictly monotone schemes. Engquist and Osher proved the stability of the first-order general monotone scheme for the scalar case [3]. Smyrlis [23] proved stability of a scalar stationary discrete shock wave for the Lax-Wendroff scheme. For scalar conservation laws, the L-F scheme belongs to the class of monotone schemes which have been well understood, see [2], [21], etc. Tadmor [25] studied the large time behavior for the rarefaction waves for some monotone schemes. The earliest important works in the study of the large time behavior for parabolic equations and monotone schemes can be seen in [5].

For the L-F scheme approximating systems, in the case that far field is a constant state, Chern [1] proved stability of the L-F scheme using diffusion waves. Liu and Xin [14] have proved that, for the L-F scheme, the solutions of the Riemann problem are single or multiple shocks; and if the summation of the initial perturbations equal zero, then the scheme solutions are asymptotically stable; they also study the stability of stationary discrete shock in [15]. The existence of discrete shock waves of first-order accurate finite difference methods for systems of conservation laws was established by Majda and Ralston [16] by using the center manifold theorem.

Our stability analysis is strongly motivated by the nonlinear stability of viscous shock profile for a viscous conservation law of the form (1.6) which is one of the hot spots in mathematical physics and interests many mathematicians (see [6], [20], [19], [9]). Studies on systems began with the independent works of Matsumura-Nishihara [17] and Goodman [4]. Important progress for general initial perturbations has been 
achieved by Liu [12] and Szepessy and Xin [24]. Recently, some interesting papers on the stability of viscous shock profiles in the case of $I(f) \neq \emptyset$, where $I(f)$ is the set of a reflection point of $f$, appeared (see [10], [8], [22], [11]).

As to the decay rate, Il'in and Oleinik [6] showed in the case of $I(f)=\emptyset$ that if the integral of the initial disturbance over $(-\infty, x]$ decays exponentially $e^{-\alpha|x|}$ (with some $\alpha>0$ ) for $|x| \rightarrow \infty$, then the solution approaches, in the maximum norm, the viscous shock profiles at an exponential rate $e^{-\beta t}$ (for some $\beta>0$ ) as $t \rightarrow \infty$. In the particular case $f=u^{2} / 2$, Nishihara [18] showed further properties, if the initial disturbance over $(-\infty, x]$ has an algebraic order $O\left(|x|^{-\alpha}\right)$ (with some $\alpha>0$ ) for $|x| \rightarrow \infty$, then the solution converges, in the maximum norm, to the shock profiles at the same algebraic rate $t^{-\alpha}$ as $t \rightarrow \infty$. He also notes that this time decay rate is optimal in general. These detailed results are not known for general $f$ with convexity. However, for such $f$, Kawashima and Matsumura [9] showed that if $\left(1+|x|^{2}\right)^{\alpha / 2} \phi_{0} \in H^{2}(\exists \alpha>0)$ is suitably small, then it holds that $\sup _{x \in R}|u-U| \leq C(1+t)^{-[\alpha] / 2}$, where $\phi_{0}$ is the integral of initial disturbance over $(-\infty, x]$ of viscous shock profile $U$. Recently, for $I(f) \neq \emptyset$ and $s \neq f^{\prime}\left(u_{ \pm}\right)$, Jones et al. [8] have shown $\sup _{R}|u-U| \leq C(1+t)^{-[\alpha] / 4}$ based on the spectral analysis. In [11] we proved that $\sup _{R}|u-U| \leq C(1+t)^{-[\alpha] / 2}$ for non-convex $f$ by introducing a weight function to overcome the difficulty caused by non-convexity of $f$.

Our main task is to estimate the time decay rate. To carry out our analysis, we use the weighted energy integration method, with regard to this method we point especially to the works [14], [15] from which we draw ideas in the present work. The specific choice of our time-dependent weight is made to insure that the information can be transferred from spatial decay to temporal decay. In its general approach,

our method resembles that of [15], but there are also essential differences between the two methods.

Our plan of this paper is as follows. After stating the notations, we reformulate the original problem and state theorems for the reformulated one. In section 3, we investigate the time decay rate when $f$ is convex, due to the weighted energy method the time-dependent weight $(1+n h)^{\gamma}\left(1+(j r)^{2}\right)^{\beta / 2}$ plays a central role in this procedure.

Notations. Let us now define the following weighted $l^{2}$ spaces,

$$
l_{K}^{2}=\left\{f_{j}: \quad\left\|f_{j}\right\|_{l_{K}^{2}} \equiv\left|f_{j}\right|_{K}=\left[\sum_{j}\left|f_{j}\right|^{2} K_{j}\right]^{\frac{1}{2}}<\infty\right\},
$$

where $K_{j}$ is a discrete weight function. When $K_{j}=\langle j r\rangle^{\alpha}=\left(1+(j r)^{2}\right)^{\frac{\alpha}{2}}$, we write $l_{K}^{2}=l_{\alpha}^{2}$ and $|\cdot|_{K}=|\cdot|_{\alpha}$; when $K_{j}=\langle j r\rangle^{\alpha} w_{j}$, we write $l_{K}^{2}=l_{\alpha, w}^{2}$ with the norm $|\cdot|_{K}=|\cdot|_{\alpha, w}$; when $C^{-1} \leq w_{j} \leq C$, we note that $l^{2}=l_{w}^{2}$ with the norm $\|\cdot\| \sim|\cdot|_{w}$ and that $l_{\alpha, w}^{2}=l_{\alpha}^{2}$ with $|\cdot|_{\alpha, w} \sim|\cdot|_{\alpha}$.

\section{Reformulation of the PRoblem}

Let $\phi_{j}$ be a stationary discrete shock wave for the L-F scheme (1.1), that is , $\phi_{j}$ satisfies (1.7a)

$$
\lambda\left(f\left(\phi_{j+1}\right)-f\left(\phi_{j-1}\right)\right)=\mu\left(\phi_{j+1}-2 \phi_{j}+\phi_{j-1}\right),
$$

summing it over $j$ from $-\infty$ to $j$ yields

$$
\lambda\left(f\left(\phi_{j+1}\right)-2 f\left(u_{ \pm}\right)+f\left(\phi_{j}\right)\right)=\mu\left(\phi_{j+1}-\phi_{j}\right),
$$


that is

$$
\mu\left(\phi_{j+1}-\phi_{j}\right)=\lambda\left(Q_{j+1}+Q_{j}\right)
$$

which has a unique solution $\phi_{j}$ up to a shift satisfying $\phi_{j}( \pm \infty)=u_{ \pm}$. We have

Lemma 2.1. Suppose (1.3a)-(1.3b) and $u_{+}<u_{-}$for $s=0$, then for each $\bar{u} \in$ $\left(u_{+}, u_{-}\right)$there exists a unique stationary discrete shock profile $\phi_{j}$ to (1.1), i.e., (1.7a)-(1.7b) holds and $\phi_{j}$ satisfies

$$
\phi_{0}=\bar{u}, \quad \phi_{j}>\phi_{j+1}, \quad \text { for } j \in Z \text {. }
$$

The proof of Lemma 2.1 is a consequence of the fact that a shock profile continuously depends on its value at a point (see [7]), we omit it here.

To obtain the decay rate, let us rewrite the initial value problem $(1.1),(1.8)$ by setting

$$
v_{j}^{n}=\sum_{k=-\infty}^{j}\left(u_{k}^{n}-\phi_{k}\right),
$$

then $v_{j}^{n} \rightarrow 0$ as $j \rightarrow \pm \infty$. Subtracting (1.7a) from (1.1), and summing up the resulting expression from $-\infty$ to $j$, we get

$$
\begin{aligned}
v_{j}^{n+1} & -v_{j}^{n}+\frac{\lambda}{2}\left(f\left(u_{j+1}^{n}\right)-f\left(\phi_{j+1}\right)\right)+\frac{\lambda}{2}\left(f\left(u_{j}^{n}\right)-f\left(\phi_{j}\right)\right) \\
& =\frac{\mu}{2}\left(v_{j+1}^{n}-2 v_{j}^{n}+v_{j-1}^{n}\right) .
\end{aligned}
$$

Noting $u_{j}^{n}-\phi_{j}=v_{j}^{n}-v_{j-1}^{n}$, we have

$$
\begin{aligned}
v_{j}^{n+1}- & v_{j}^{n}+\frac{\lambda}{2} f^{\prime}\left(\phi_{j+1}^{n}\right)\left(v_{j+1}^{n}-v_{j}^{n}\right)+\frac{\lambda}{2} f^{\prime}\left(\phi_{j}^{n}\right)\left(v_{j}^{n}-v_{j-1}^{n}\right) \\
& +\frac{\lambda}{2} F\left(\phi_{j+1}^{n}, v_{j+1}^{n}-v_{j}^{n}\right)+\frac{\lambda}{2} F\left(\phi_{j}^{n}, v_{j}^{n}-v_{j-1}^{n}\right)=\frac{\mu}{2}\left(v_{j+1}^{n}-2 v_{j}^{n}+v_{j-1}^{n}\right),
\end{aligned}
$$

where

$$
F(\phi, u-\phi)=f(u)-f(\phi)-f^{\prime}(\phi)(u-\phi),
$$

satisfies the estimate

$$
|F(\phi, u-\phi)| \leq O(1)|u-\phi|^{2}
$$

here $O(1)$ is a positive constant. Using the notations

$$
\Lambda_{j}=f^{\prime}\left(\phi_{j}\right), \quad \theta_{j}^{n}=F\left(\phi_{j}, v_{j}^{n}-v_{j-1}^{n}\right),
$$

we may rewrite the equation (2.4) as

$$
\begin{aligned}
v_{j}^{n+1}- & v_{j}^{n}+\frac{\lambda}{2} \Lambda_{j+1}\left(v_{j+1}^{n}-v_{j}^{n}\right)+\frac{\lambda}{2} \Lambda_{j}\left(v_{j}^{n}-v_{j-1}^{n}\right) \\
-\frac{\mu}{2}\left(v_{j+1}^{n}-2 v_{j}^{n}+v_{j-1}^{n}\right) & =e_{j}^{n},
\end{aligned}
$$

where

$$
e_{j}^{n}=-\frac{\lambda}{2}\left(\theta_{j+1}^{n}+\theta_{j}^{n}\right)
$$

Noting that (1.12) implies $\left|v_{j}^{0}\right|_{\alpha}$ is suitably small (which will be shown in the next section), then the problem (2.6) with initial data $v_{j}^{0}$ can be solved globally in time as follows. 
Theorem 2.1. Suppose $v_{j}^{0} \in l_{\alpha}^{2}$ for some $\alpha \geq 0$ and $f^{\prime \prime}>0$, then there exists a positive constant $\delta_{2}$ such that if $\left|v_{j}^{0}\right|_{\alpha}<\delta_{2}$, the problem (2.6) with initial data $v_{j}^{0}$ has a unique global solution $v_{j}^{n}$ satisfying, for any $n \geq 0$

$$
\sup _{n}(1+n h)^{\gamma}\left\|v_{j}^{n}\right\|^{2}+\sum_{n}(1+n h)^{\gamma}\left\|v_{j+1}^{n}-v_{j}^{n}\right\|^{2} \leq C\left|v_{j}^{0}\right|_{\alpha}^{2}
$$

for any $\gamma$ such that $0 \leq \gamma \leq \alpha$ if $\alpha$ is an integer and that $0 \leq \gamma<\alpha$ if $\alpha$ is not an integer.

From (2.6), $v_{j}^{n+1}$ can be expressed in terms of $v_{j}^{n}$ in the explicit scheme, we can obtain $v_{j}^{n}$ step by step from the beginning of $n=0$. Moreover, we can estimate the $l^{2}$-norm of $v_{j}^{n}$ as follows

$$
\sum_{j}\left(v_{j}^{n+1}\right)^{2} \leq C \sum_{j}\left(v_{j}^{n}\right)^{2}
$$

Combining (2.8) with the following a priori estimate and a standard continuity argument proves that Theorem 2.1 holds.

Proposition 2.2 (A priori estimate). Let $n_{1}$ be a positive integer. Suppose that the problem (2.6) with initial data $v_{j}^{0}$ has a unique solution $v_{j}^{n} \in l_{\alpha}^{2}$ for some $\alpha \geq 0$, then $v_{j}^{n}$ satisfies (2.7) for $0 \leq n \leq n_{1}$, provided $\sup _{0 \leq n \leq n_{1}}\left\|v_{j}^{n}\right\|$ is suitably small.

The global existence can be proved in a way similar to that in [13]; we omit the details. For the proofs of (2.7) more estimates are necessary.

\section{Time-DeCAy estimates}

We proceed with more a priori estimates of the solution $v_{j}^{n}$ of the problem (2.6) with initial data $v_{j}^{0}$. In order to estimate the time-decay rate, we introduce a timedependent discrete weight function $K_{j}^{n}$, then multiplying (2.6) by $2 v_{j}^{n} K_{j}^{n}$ and summing over $j$, we obtain

$$
\begin{gathered}
\sum_{j} 2\left(v_{j}^{n+1}-v_{j}^{n}\right) v_{j}^{n} K_{j}^{n}+\lambda\left\{\sum_{j} \Lambda_{j+1} v_{j}^{n} K_{j}^{n}\left(v_{j+1}^{n}-v_{j}^{n}\right)+\sum_{j} \Lambda_{j} v_{j}^{n} K_{j}^{n}\left(v_{j}^{n}-v_{j-1}^{n}\right)\right\} \\
+\mu \sum_{j} v_{j}^{n} K_{j}^{n}\left(2 v_{j}^{n}-v_{j+1}^{n}-v_{j-1}^{n}\right)=2 \sum_{j} v_{j}^{n} K_{j}^{n} e_{j}^{n} .
\end{gathered}
$$


We now estimate each term denoted by $I_{i}(i=1,2,3)$ in the sequel on the left-hand side of (3.1), we have

$$
\begin{aligned}
I_{1}= & \sum_{j}\left\{\left(v_{j}^{n+1}\right)^{2}-\left(v_{j}^{n+1}-v_{j}^{n}\right)^{2}-\left(v_{j}^{n}\right)^{2}\right\} K_{j}^{n} \\
= & \sum_{j}\left(v_{j}^{n+1}\right)^{2} K_{j}^{n+1}-\sum_{j}\left(v_{j}^{n}\right)^{2} K_{j}^{n} \\
& -\sum_{j}\left(v_{j}^{n+1}-v_{j}^{n}\right)^{2} K_{j}^{n}-\sum_{j}\left(v_{j}^{n+1}\right)^{2}\left(K_{j}^{n+1}-K_{j}^{n}\right), \\
I_{2}= & \lambda\left\{\sum_{j} \Lambda_{j+1} K_{j}^{n} v_{j}^{n} v_{j+1}^{n}-\sum_{j} \Lambda_{j+1} K_{j}^{n}\left(v_{j}^{n}\right)^{2}\right. \\
& \left.+\sum_{j} \Lambda_{j} K_{j}^{n}\left(v_{j}^{n}\right)^{2}-\sum_{j} \Lambda_{j} K_{j}^{n} v_{j}^{n} v_{j-1}^{n}\right\} \\
= & \lambda\left\{-\sum_{j}\left(\Lambda_{j+1} K_{j+1}^{n}-\Lambda_{j} K_{j}^{n}\right)\left(v_{j}^{n}\right)^{2}\right. \\
& \left.+\sum_{j} \Lambda_{j+1}\left(K_{j}^{n}-K_{j+1}^{n}\right) v_{j}^{n}\left(v_{j+1}^{n}-v_{j}^{n}\right)\right\}, \\
& \left.+\sum_{j}\left(K_{j+1}^{n}-K_{j}^{n}\right)_{j+1}^{n}+v_{j}^{n}\left(v_{j+1}^{n}-v_{j}^{n}\right)\right\} . \\
I_{3}= & \mu\left\{\sum_{j} v_{j}^{n} K_{j}^{n}\left(v_{j}^{n}-v_{j+1}^{n}\right)-\sum_{j} v_{j}^{n} K_{j}^{n}\left(v_{j-1}^{n}-v_{j}^{n}\right)\right\} \\
= & \mu\left\{\sum_{j} \frac{K_{j}^{n}}{2}\left[\left(v_{j}^{n}-v_{j+1}^{n}\right)^{2}+\left(v_{j}^{n}\right)^{2}-\left(v_{j+1}^{n}\right)^{2}\right]\right. \\
& \left.+\sum_{j} \frac{K_{j+1}^{n}}{2}\left[\left(v_{j}^{n}-v_{j+1}\right)^{2}+\left(v_{j+1}^{n}\right)^{2}-\left(v_{j}^{n}\right)^{2}\right]\right\} \\
& \left.v_{j+1}^{n}\right)^{2} \frac{K_{j}^{n}+K_{j+1}^{n}}{2} \\
& \\
& \\
& \\
&
\end{aligned}
$$

Then we have

$$
\begin{aligned}
& \sum_{j}\left(v_{j}^{n+1}\right)^{2} K_{j}^{n+1}-\sum_{j}\left(v_{j}^{n}\right)^{2} K_{j}^{n}+\sum_{j} A_{j}^{n}\left(v_{j}^{n}\right)^{2}+\mu \sum_{j} \frac{K_{j}^{n}+K_{j+1}^{n}}{2}\left|v_{j+1}^{n}-v_{j}^{n}\right|^{2} \\
& =\sum_{j}\left(v_{j}^{n+1}-v_{j}^{n}\right)^{2} K_{j}^{n}-\sum_{j} B_{j}^{n}+\sum_{j}\left(K_{j}^{n+1}-K_{j}^{n}\right)\left(v_{j}^{n+1}\right)^{2}+2 \sum_{j} v_{j}^{n} K_{j}^{n} e_{j}^{n},
\end{aligned}
$$


where

$$
\begin{aligned}
A_{j}^{n} & =-\lambda\left(\Lambda_{j+1} K_{j+1}^{n}-\Lambda_{j} K_{j}^{n}\right), \\
B_{j}^{n} & =\left(-\lambda \Lambda_{j+1} v_{j}^{n}+\mu \frac{v_{j+1}^{n}+v_{j}^{n}}{2}\right)\left(K_{j}^{n}-K_{j+1}^{n}\right)\left(v_{j+1}^{n}-v_{j}^{n}\right) .
\end{aligned}
$$

Now setting

$$
K_{j}^{n}=(1+n h)^{\gamma}\langle j r\rangle^{\beta}
$$

and denoting $\left|v_{j}^{n}\right|_{\beta}^{2}=\sum_{j}\langle j r\rangle^{\beta}\left|v_{j}^{n}\right|^{2}$. Then

$$
\frac{K_{j}^{n}+K_{j+1}^{n}}{2}=(1+n h)^{\gamma} \frac{\langle j r\rangle^{\beta}+\langle(j+1) r\rangle^{\beta}}{2} \geq(1+n h)^{\gamma}\langle j r\rangle^{\beta},
$$

and

$$
\begin{aligned}
K_{j}^{n+1}-K_{j}^{n} & =\langle j r\rangle^{\beta}\left((1+(n+1) h)^{\gamma}-(1+n h)^{\gamma}\right) \\
& =\langle j r\rangle^{\beta}\left(\gamma(1+n h)^{\gamma-1} h+\frac{\gamma(\gamma-1)(1+\tilde{n} h)^{\gamma-2}}{2} h^{2}\right) \\
& =\gamma(1+O(h))\langle j r\rangle^{\beta}(1+n h)^{\gamma-1} h, \quad(n<\tilde{n}<n+1),
\end{aligned}
$$

which leads to

$$
\begin{aligned}
\sum_{j}\left(K_{j}^{n+1}-K_{j}^{n}\right)\left(v_{j}^{n+1}\right)^{2} \leq 2 \gamma(1+O(h))\left\{(1+n h)^{\gamma-1} \sum_{j}\langle j r\rangle^{\beta}\left(v_{j}^{n}\right)^{2} h\right. \\
\quad+\frac{h}{1+n h}\left((1+n h)^{\gamma} \sum_{j}\langle j r\rangle^{\beta}\left(v_{j}^{n+1}-v_{j}^{n}\right)^{2}\right\} \\
\leq C \gamma(1+n h)^{\gamma-1}\left|v_{j}^{n}\right|_{\beta}^{2} h+O(1) h(1+n h)^{\gamma}\left|v_{j}^{n+1}-v_{j}^{n}\right|_{\beta}^{2},
\end{aligned}
$$

where we have used

$$
\left(v_{j}^{n+1}\right)^{2}=\left(v_{j}^{n+1}-v_{j}^{n}+v_{j}^{n}\right)^{2} \leq 2\left(v_{j}^{n+1}-v_{j}^{n}\right)^{2}+2\left(v_{j}^{n}\right)^{2} .
$$

Collecting (3.2), (3.5) and (3.6) we have

$$
\begin{aligned}
(1+(n+1) h)^{\gamma}\left|v_{j}^{n+1}\right|_{\beta}^{2}-(1+n h)^{\gamma}\left|v_{j}^{n}\right|_{\beta}^{2} & \\
& +\alpha(1+n h)^{\gamma}\left|v_{j+1}^{n}-v_{j}^{n}\right|_{\beta}^{2}+\sum_{j} A_{j}^{n}\left(v_{j}^{n}\right)^{2} \\
\leq & (1+O(1) h)(1+n h)^{\gamma}\left|v_{j}^{n+1}-v_{j}^{n}\right|_{\beta}^{2}+\sum_{j}\left|B_{j}^{n}\right| \\
& +C_{1} \gamma(1+n h)^{\gamma-1} h\left|v_{j}^{n}\right|_{\beta}^{2}+2 \sum_{j} v_{j}^{n} K_{j}^{n} e_{j}^{n} .
\end{aligned}
$$

Next we estimate the terms on the right-hand side of (3.7), we set

$$
N\left(n_{1}\right)=\sup _{n \leq n_{1}}\left(\sum_{j}\left|v_{j}^{n}\right|^{2}\right)^{1 / 2},
$$

and assume that $N\left(n_{1}\right)$ is small. Obviously, we have

$$
\sup _{n \leq n_{1}, j}\left|v_{j}^{n}\right| \leq N\left(n_{1}\right) \text {. }
$$


It follows from equation (2.6) that

$$
v_{j}^{n+1}-v_{j}^{n}=\left\{\frac{\mu}{2}-\frac{\lambda}{2} \Lambda_{j+1}\right\}\left(v_{j+1}^{n}-v_{j}^{n}\right)-\left\{\frac{\mu}{2}+\frac{\lambda}{2} \Lambda_{j}\right\}\left(v_{j}^{n}-v_{j-1}^{n}\right)+e_{j}^{n}
$$

then we have

$$
\begin{aligned}
\mid v_{j}^{n+1}- & \left.v_{j}^{n}\right|^{2} \leq \frac{1}{2}\left\{\left(\mu-\lambda \Lambda_{j+1}\right)^{2}\left|v_{j+1}^{n}-v_{j}^{n}\right|^{2}+\left(\mu+\lambda \Lambda_{j}\right)^{2}\left|v_{j}^{n}-v_{j-1}^{n}\right|^{2}\right\} \\
& +O(1) N\left(n_{1}\right)\left\{\left|v_{j+1}^{n}-v_{j}^{n}\right|^{2}+\left|v_{j}^{n}-v_{j-1}^{n}\right|^{2}\right\}
\end{aligned}
$$

where we have used (2.5b) and (3.9). Consequently, we have

$$
\sum_{j}\langle j r\rangle^{\beta}\left|v_{j}^{n+1}-v_{j}^{n}\right|^{2} \leq\left(\left(\mu+\lambda \max \left|f^{\prime}\right|\right)^{2}+O(1) N\left(n_{1}\right)\right) \times\left|v_{j+1}^{n}-v_{j}^{n}\right|_{\beta}^{2} .
$$

Next using (2.5b) and (3.9), we get

$$
2 \sum_{j}\left|v_{j}^{n} K_{j}^{n} e_{j}^{n}\right| \leq C N\left(n_{1}\right)(1+n h)^{\gamma} \sum_{j}\langle j r\rangle^{\beta}\left|v_{j+1}^{n}-v_{j}^{n}\right|^{2} .
$$

To get the desired estimate, we must estimate $\sum_{j} A_{j}^{n}\left(v_{j}^{n}\right)^{2}$ and $\sum_{j} B_{j}^{n}$.

Step 1. We estimate $\sum_{j} A_{j}^{n}\left(v_{j}^{n}\right)^{2}$. For the estimate, we need some properties of the stationary discrete shock wave. Let $u^{*}$ be the unique state determined by

$$
0=\frac{f\left(u_{+}\right)-f\left(u_{-}\right)}{u_{+}-u_{-}}=f^{\prime}\left(u^{*}\right)
$$

since the discrete shock profile continuously depends on its value at a point and $\phi_{j}$ is strictly decreasing in $j \in Z$. On the other hand, the uniqueness of the shock profile is understood modulo translation; without loss of generality, we assume $\phi_{0}=u^{*}$. Thus $\Lambda_{0}=Q^{\prime}\left(\phi_{0}\right)=0$ and $Q^{\prime}\left(\phi_{-1}\right)>0>Q^{\prime}\left(\phi_{1}\right)$ due to $Q^{\prime \prime}(u)>0$.

With respect to $A_{j}^{n}$ we have the following lemma.

Lemma 3.1. For any $\beta \in[0, \alpha]$, there is a positive constant $c_{0}$ independent of $\beta$ such that

$$
A_{j}^{n} \geq c_{0} \beta(1+n h)^{\gamma}\langle j r\rangle^{\beta-1} h,
$$

for any $j \in Z$, provided that $\lambda$ is suitably small.

Proof. By the Taylor expression formula we have

$$
\begin{aligned}
\langle(j+1) r\rangle^{\beta}= & \langle j r\rangle^{\beta}+\beta j r\langle j r\rangle^{\beta-2} r \\
& +\beta\langle\tilde{j} r\rangle^{\beta-4}\left(\langle\tilde{j} r\rangle^{2}+(\beta-2)(\tilde{j} r)^{2}\right) r^{2} / 2
\end{aligned}
$$

and

$$
\Lambda_{j+1}=Q^{\prime}\left(\phi_{j+1}\right)=Q^{\prime}\left(\phi_{j}\right)+Q^{\prime \prime}\left(\bar{\phi}_{j}\right)\left(\phi_{j+1}-\phi_{j}\right) .
$$


Together with (3.14) and (3.15), we have

$$
\begin{aligned}
A_{j}^{n}= & -\lambda(1+n h)^{\gamma}\left[\langle(j+1) r\rangle^{\beta} \Lambda_{j+1}-\langle j r\rangle^{\beta} \Lambda_{j}\right] \\
= & -\lambda(1+n h)^{\gamma}\left[\beta j r\langle j r\rangle^{\beta-2} Q^{\prime}\left(\phi_{j}\right) r\right. \\
& \left.+Q^{\prime \prime}\left(\bar{\phi}_{j}\right)\langle j r\rangle^{\beta}\left(\phi_{j+1}-\phi_{j}\right)+\langle j r\rangle^{\beta-1} O(1) r^{2}\right] \\
= & (1+n h)^{\gamma}\langle j r\rangle^{\beta-1} h\left[-\beta \frac{j r}{\langle j r\rangle} Q^{\prime}\left(\phi_{j}\right)-\langle j r\rangle Q^{\prime \prime}\left(\bar{\phi}_{j}\right) \frac{\phi_{j+1}-\phi_{j}}{r}+O(r)\right],
\end{aligned}
$$

where we have used $\left|\phi_{j+1}-\phi_{j}\right|=\left|\frac{\phi_{j+1}-\phi_{j}}{r}\right| r \leq O(1) r$ and $\lambda r=h$.

Due to (3.15), we have $Q^{\prime}\left(\phi_{j+1}\right)-Q^{\prime}\left(\phi_{j}\right)<0$, by virtue of $f^{\prime}\left(u_{+}\right)<0<f^{\prime}\left(u_{-}\right)$, so $-\frac{j r}{\langle j r\rangle} Q^{\prime}\left(\phi_{j}\right) \rightarrow \mp Q^{\prime}\left(u_{ \pm}\right)=\mp f^{\prime}\left(u_{ \pm}\right)>0$, as $j \rightarrow \pm \infty$, so

$$
-\frac{j r}{\langle j r\rangle} Q^{\prime}\left(\phi_{j}\right) \geq c, \quad j \neq 0
$$

for some $c>0$. On the other hand,

$$
-\langle j r\rangle Q^{\prime \prime}\left(\bar{\phi}_{j}\right) \frac{\phi_{j+1}-\phi_{j}}{r}=\frac{\phi_{0}-\phi_{1}}{r} Q^{\prime \prime}\left(\bar{\phi}_{0}\right)=c_{1}>0, \quad j=0 .
$$

Combining (3.17) with (3.18), we obtain (3.13), where $c_{0}>\min \left\{c, \frac{c_{1}}{\alpha}\right\}$, provided $\lambda$ is suitably small.

Step 2. We estimate $\sum_{j}\left|B_{j}^{n}\right|$.

First we compute

$$
\begin{aligned}
K_{j+1}^{n}-K_{j}^{n} & =(1+n h)^{\gamma}\left(\langle(j+1) r\rangle^{\beta}-\langle j r\rangle^{\beta}\right) \\
& =(1+n h)^{\gamma} \beta \tilde{j} r\langle\tilde{j} r\rangle^{\beta-2} r \\
& \leq \beta(1+n h)^{\gamma}\langle\tilde{j} r\rangle^{\beta-1} r \\
& \leq \beta c_{r}(1+n h)^{\gamma}\langle j r\rangle^{\beta-1} r,
\end{aligned}
$$

where $c_{r}=c_{r, \beta}=\sup _{j} \frac{\langle\tilde{j} r\rangle^{\beta-1}}{\langle j r\rangle^{\beta-1}} \quad(j<\tilde{j}<j+1)$,

$$
\begin{aligned}
& {\left[-\lambda \Lambda_{j+1} v_{j}^{n}+\mu \frac{v_{j}^{n}+v_{j+1}^{n}}{2}\right]\left(v_{j+1}^{n}-v_{j}^{n}\right)} \\
& \quad=\left[\left(\mu-\lambda \Lambda_{j+1}\right) v_{j}^{n}+\frac{\mu}{2}\left(v_{j+1}^{n}-v_{j}^{n}\right)\right]\left(v_{j+1}^{n}-v_{j}^{n}\right) \\
& \quad \leq \frac{\left(\mu+\lambda \max \left|f^{\prime}\right|\right)^{2}}{4 \varepsilon}\left|v_{j}^{n}\right|^{2}+\left(\varepsilon+\frac{\mu}{2}\right)\left|v_{j+1}^{n}-v_{j}^{n}\right|^{2}
\end{aligned}
$$


for any $\varepsilon>0$. Combining (3.3), (3.19) with (3.20), we obtain

$$
\begin{aligned}
\sum_{j}\left|B_{j}^{n}\right| \leq & c_{r} \beta(1+n h)^{\gamma} \sum_{j}\langle j r\rangle^{\beta-1} \\
& \times\left[\frac{\left(\mu+\lambda \max \left|f^{\prime}\right|\right)^{2}}{4 \varepsilon}\left|v_{j}^{n}\right|^{2}+\left(\varepsilon+\frac{\mu}{2}\right)\left|v_{j+1}^{n}-v_{j}^{n}\right|^{2}\right] r \\
\leq & \beta(1+n h)^{\gamma}\left[\frac{c_{r}\left(\mu+\lambda \max \left|f^{\prime}\right|\right)^{2}}{4 \varepsilon} r\left|v_{j}^{n}\right|_{\beta-1}^{2}\right. \\
+ & \left.c_{r}\left(\varepsilon+\frac{\mu}{2}\right)\left|v_{j+1}^{n}-v_{j}^{n}\right|_{\beta-1}^{2} r\right] .
\end{aligned}
$$

But

$$
\begin{aligned}
\left|v_{j+1}^{n}-v_{j}^{n}\right|_{\beta-1}^{2} & =\sum_{|j| \leq J}\langle j r\rangle^{\beta-1}\left|v_{j+1}^{n}-v_{j}^{n}\right|^{2}+\sum_{|j| \geq J} \frac{\langle j r\rangle^{\beta}}{\langle j r\rangle}\left|v_{j+1}^{n}-v_{j}^{n}\right|^{2} \\
& \leq C(J) \sum_{j}\left|v_{j+1}^{n}-v_{j}^{n}\right|^{2}+\frac{1}{J r} \sum_{j}\langle j r\rangle^{\beta}\left|v_{j+1}^{n}-v_{j}^{n}\right|^{2} \\
& \leq C(J)\left\|v_{j+1}^{n}-v_{j}^{n}\right\|^{2}+\frac{1}{J r}\left|v_{j+1}^{n}-v_{j}^{n}\right|_{\beta}^{2}
\end{aligned}
$$

for some large fixed number $J>0$, we have

$$
\begin{aligned}
\sum_{j}\left|B_{j}^{n}\right| \leq & \beta(1+n h)^{\gamma}\left[\frac{c_{0} h}{2}\left|v_{j}^{n}\right|_{\beta-1}^{2}\right. \\
& \left.+C(J) c_{r}\left(\varepsilon+\frac{\mu}{2}\right) r \| v_{j+1}^{n}-\left.v_{j}^{n}\right|^{2}+\frac{c_{r}(2 \varepsilon+\mu)}{2 J}\left|v_{j+1}^{n}-v_{j}^{n}\right|_{\beta}^{2}\right] \\
\leq & \frac{c_{0} \beta h}{2}(1+n h)^{\gamma}\left|v_{j}^{n}\right|_{\beta-1}^{2}+C \beta(1+n h)^{\gamma}|| v_{j+1}^{n}-\left.v_{j}^{n}\right|^{2} \\
& +\frac{\beta c_{r}(2 \varepsilon+\mu)}{2 J}(1+n h)^{\gamma}\left|v_{j+1}^{n}-v_{j}^{n}\right|_{\beta}^{2},
\end{aligned}
$$

here we have chosen

$$
\varepsilon \geq \frac{c_{r}\left(\mu+\lambda \max \left|f^{\prime}\right|\right)^{2}}{2 c_{0} \lambda}
$$

and $C \geq \max \left\{C(J) c_{r}\left(\varepsilon+\frac{\alpha}{2}\right) r, C_{1}\right\}$.

Assuming Lemma 3.1, we obtain the following basic a priori estimate:

Proposition 3.2. Let $v_{j}^{n}$ be a solution of (2.6) for $n \leq n_{1}$. Then there exists a positive constant $C$ independent of $n_{1}$ such that for all $n \leq n_{1}$

$$
\begin{aligned}
& (1+n h)^{\gamma}\left|v_{j}^{n}\right|_{\beta}^{2}+\beta \sum_{i<n}(1+i h)^{\gamma}\left|v_{j}^{i}\right|_{\beta-1}^{2} h+\sum_{i<n}(1+i h)^{\gamma}\left|v_{j+1}^{i}-v_{j}^{i}\right|_{\beta}^{2} \\
& \leq C\left\{\left|v_{j}^{0}\right|_{\beta}^{2}+\gamma \sum_{i<n}(1+i h)^{\gamma-1}\left|v_{j}^{i}\right|_{\beta}^{2} h+\beta \sum_{i<n}(1+i h)^{\gamma}|| v_{j+1}^{i}-v_{j}^{i}||^{2}\right\},
\end{aligned}
$$

provided $\lambda$ and $N\left(n_{1}\right)$ are suitably small. 
Proof. By Lemma 3.1, we collect (3.7), (3.11)-(3.13) and (3.22) to obtain

$$
\begin{aligned}
(1+( & +1) h)^{\gamma}\left|v_{j}^{n+1}\right|_{\beta}^{2}-(1+n h)^{\gamma}\left|v_{j}^{n}\right|_{\beta}^{2} \\
& +\left\{\mu-(1+O(h))\left[\left(\mu+\lambda \max \left|f^{\prime}\right|\right)^{2}+O(1) N\left(n_{1}\right)\right]-C N\left(n_{1}\right)\right. \\
& \left.-\frac{\beta c_{r}(2 \varepsilon+\mu)}{2 J}\right\}(1+n h)^{\gamma}\left|v_{j+1}^{n}-v_{j}^{n}\right|_{\beta}^{2}+\frac{c_{0} \beta h}{2}(1+n h)^{\gamma}\left|v_{j}^{n}\right|_{\beta-1}^{2} \\
\leq & C\left\{\beta(1+n h)^{\gamma}|| v_{j+1}^{n}-v_{j}^{n} \|^{2}+\gamma(1+n h)^{\gamma-1} h\left|v_{j}^{n}\right|_{\beta}^{2}\right\},
\end{aligned}
$$

here we have used $A_{j}^{n} \geq c_{0} \beta h(1+n h)^{\gamma}\langle j r\rangle^{\beta-1}$. On one hand, since $\mu<1$, we take suitably small $\lambda$ and take $J$ suitably large, then

$$
\begin{gathered}
\mu-(1+O(h))\left[\left(\mu+\lambda \max \left|f^{\prime}\right|\right)^{2}-O(1) N\left(n_{1}\right)\right] \\
-C N\left(n_{1}\right)-\frac{\beta c_{r}(2 \varepsilon+\mu)}{2 J}>\nu,
\end{gathered}
$$

here $0<\nu<\mu$, provided $N\left(n_{1}\right)$ is suitably small. Finally, summing the two sides of (3.24) from 0 to $n-1$ with respect to $n$, by virtue of (3.25), we have

$$
\begin{gathered}
(1+n h)^{\gamma}\left|v_{j}^{n}\right|_{\beta}^{2}+\nu \sum_{i<n}(1+i h)^{\gamma}\left|v_{j+1}^{i}-v_{j}^{i}\right|_{\beta}^{2}+\frac{c_{0} \beta}{2} \sum_{i<n}(1+i h)^{\gamma}\left|v_{j}^{i}\right|_{\beta-1}^{2} h \\
\leq C\left\{\left|v_{j}^{0}\right|_{\beta}^{2}+\beta \sum_{i<n}(1+i h)^{\gamma}|| v_{j+1}^{i}-\left.v_{j}^{i}\right|^{2}+\gamma \sum_{i<n}(1+i h)^{\gamma-1}\left|v_{j}^{i}\right|_{\beta}^{2} h\right\},
\end{gathered}
$$

then (3.23) follows immediately.

We proceed to estimate the solution of the problem (2.6). First, taking $\beta=\gamma=0$ in (3.23), it is easy to obtain the following lemma:

Lemma 3.3. There is a positive constant $C$ independent of $n_{1}$, it holds for $n \in$ $\left[0, n_{1}\right]$ that

$$
\left\|v_{j}^{n}\right\|^{2}+\sum_{i<n}\left\|v_{j+1}^{i}-v_{j}^{i}\right\|^{2} \leq C\left\|v_{j}^{0}\right\|^{2},
$$

provided $N\left(n_{1}\right)$ and $\lambda$ are suitably small.

Applying the induction to (3.23) we have

Lemma 3.4. Let $\gamma \in[0, \alpha]$ be an integer. Then it holds for $n \in\left[0, n_{1}\right]$ that

$$
\begin{gathered}
(1+n h)^{\gamma}\left|v_{j}^{n}\right|_{\alpha-\gamma}^{2}+(\alpha-\gamma) \sum_{i<n}(1+i h)^{\gamma}\left|v_{j}^{i}\right|_{\alpha-\gamma-1}^{2} h \\
+\sum_{i<n}(1+i h)^{\gamma}\left|v_{j+1}^{i}-v_{j}^{i}\right|_{\alpha-\gamma}^{2} \leq C\left|v_{j}^{0}\right|_{\alpha}^{2} .
\end{gathered}
$$

Consequently, if $\alpha$ is an integer, then the following estimate holds for $0 \leq \gamma \leq \alpha$

$$
(1+n h)^{\gamma}\left\|v_{j}^{n}\right\|^{2}+\sum_{i<n}(1+i h)^{\gamma}\left\|v_{j+1}^{i}-v_{j}^{i}\right\|^{2} \leq C\left|v_{j}^{0}\right|_{\alpha}^{2} .
$$

Similar to the argument in continuous counterpart in [16], we prove this lemma as follows. 
Proof. Step 1. We take $0 \leq \alpha<1$, letting $\beta=\alpha$ and $\gamma=0$ in (3.23), we have

$$
\begin{gathered}
\left|v_{j}^{n}\right|_{\alpha}^{2}+\alpha \sum_{i<n}\left|v_{j}^{i}\right|_{\alpha-1}^{2} h+\sum_{i<n}\left|v_{j+1}^{i}-v_{j}^{i}\right|_{\alpha}^{2} \\
\leq C\left\{\left|v_{j}^{0}\right|_{\alpha}^{2}+\alpha \sum_{i<n}|| v_{j+1}^{i}-\left.v_{j}^{i}\right|^{2}\right\} .
\end{gathered}
$$

Due to $(3.27)$,

$$
\sum_{i<n}\left\|v_{j+1}^{i}-v_{j}^{i}\right\|^{2} \leq C|| v_{j}^{0} \|^{2} \leq C\left|v_{j}^{0}\right|_{\alpha}^{2}, \quad \text { for } \quad \alpha \geq 0
$$

combining this with (3.30) we obtain (3.28) with $\gamma=0$. Therefore Lemma 3.4 is proved for $0 \leq \alpha<1$.

Step 2. we take $1 \leq \alpha<2$. First, letting $\beta=0$ and $\gamma=1$ in (3.23), we have

$$
(1+n h)\left|v_{j}^{n}\right|_{0}^{2}+\sum_{i<n}(1+i h)\left|v_{j+1}^{i}-v_{j}^{i}\right|_{0}^{2} \leq C\left\{\left|v_{j}^{0}\right|_{0}^{2}+\sum_{i<n}\left|v_{j}^{i}\right|_{0}^{2} h\right\},
$$

and with (3.28) $(\gamma=0)$ to obtain (3.29) with $\gamma=1$, where we have used $\left|v_{j}^{n}\right|_{0}^{2} \leq$ $\left|v_{j}^{0}\right|_{\alpha-1}^{2} \leq\left|v_{j}^{0}\right|_{\alpha}^{2}$ for $1 \leq \alpha<2$. Secondly, letting $\beta=\alpha-1$ and $\gamma=1$ in (3.23), we have

$$
\begin{gathered}
(1+n h)\left|v_{j}^{n}\right|_{\alpha-1}^{2}+(\alpha-1) \sum_{i<n}(1+i h)\left|v_{j}^{i}\right|_{\alpha-1-1}^{2} h+\sum_{i<n}(1+i h)\left|v_{j+1}^{i}-v_{j}^{i}\right|_{\alpha-1}^{2} \\
\leq C\left\{\left|v_{j}^{0}\right|_{\alpha-1}^{2}+\sum_{i<n}\left|v_{j}^{i}\right|_{\alpha-1}^{2} h+(\alpha-1) \sum_{i<n}(1+i h)\left\|v_{j+1}^{i}-v_{j}^{i}\right\|^{2}\right\}
\end{gathered}
$$

together with (3.29) with $\gamma=1$ and (3.28) $(\gamma=0)$ to obtain (3.28) with $\gamma=1$. Therefore the proof is completed for $\alpha<2$.

Step 3. We repeat the same procedure as in Step 2. The estimate (3.23) (with $\beta=0, \gamma=2)$ together with (3.28) $(\gamma=1)$ yields (3.29) (with $\gamma=2)$, where $\alpha \geq 2$ is assumed. Also, (3.23) (with $\beta=\alpha-2, \gamma=2)$ together with $(3.28)(\gamma=1)$ and (3.29) (with $\gamma=2$ ) yields (3.28) (with $\gamma=2$ ), which proves the lemma for $\alpha<3$.

Repeating the same procedure, we can get the desired estimate (3.28) for any $\alpha \geq 0$. This completes the proof of Lemma 3.4.

Further we show sharper estimate. Let $\alpha$ be not an integer and $\gamma$ be $[\alpha]<\gamma<\alpha$. Taking $\beta=0$ in (3.23) we have

$$
\begin{array}{r}
(1+n h)^{\gamma}\left|v_{j}^{n}\right|_{0}^{2}+\sum_{i<n}(1+i h)^{\gamma}\left|v_{j+1}^{i}-v_{j}^{i}\right|_{0}^{2} \\
\leq C\left\{\left|v_{j}^{0}\right|_{0}^{2}+\gamma \sum_{i<n}(1+i h)^{\gamma-1}\left|v_{j}^{i}\right|_{0}^{2} h\right\} .
\end{array}
$$

Using (3.28) with $\gamma=[\alpha]$,

$$
\begin{gathered}
(1+n h)^{[\alpha]}\left|v_{j}^{n}\right|_{\alpha-[\alpha]}^{2}+(\alpha-[\alpha]) \sum_{i<n}(1+i h)^{[\alpha]}\left|v_{j}^{i}\right|_{\alpha-[\alpha]-1}^{2} h \\
+\sum_{i<n}(1+i h)^{[\alpha]}\left|v_{j+1}^{i}-v_{j}^{i}\right|_{\alpha-[\alpha]}^{2} \leq C\left|v_{j}^{0}\right|_{\alpha}^{2}
\end{gathered}
$$


we estimate the final term in (3.31):

$$
\begin{aligned}
& \sum_{i<n}(1+i h)^{\gamma-1}\left|v_{j}^{i}\right|_{0}^{2} h \\
& =\sum_{i<n}(1+i h)^{\gamma-1} \sum_{j}\langle j r\rangle^{(\alpha-[\alpha])([\alpha]+1-\alpha)-(\alpha-[\alpha])([\alpha]+1-\alpha)}\left(\left|v_{j}^{i}\right|^{2}\right)^{([\alpha]+1-\alpha)+(\alpha-[\alpha])} h \\
& \leq \sum_{i<n}(1+i h)^{\gamma-1}\left(\sum_{j}\langle j r\rangle^{\alpha-[\alpha]}\left|v_{j}^{i}\right|^{2}\right)^{[\alpha]+1-\alpha}\left(\sum_{j}\langle j r\rangle^{-([\alpha]+1-\alpha)}\left|v_{j}^{i}\right|^{2}\right)^{\alpha-[\alpha]} h \\
& =\sum_{i<n}(1+i h)^{-([\alpha]+1-\gamma)}\left((1+i h)^{[\alpha]}\left|v_{j}^{i}\right|_{\alpha-[\alpha]}^{2}\right)^{[\alpha]+1-\alpha}\left((1+i h)^{[\alpha]}\left|v_{j}^{i}\right|_{\alpha-[\alpha]-1}^{2}\right)^{\alpha-[\alpha]} h,
\end{aligned}
$$

where we have used the Hölder inequality

$$
\sum a b \leq\left(\sum a^{p}\right)^{1 / p}\left(\sum b^{p^{\prime}}\right)^{1 / p^{\prime}}, \quad \frac{1}{p}+\frac{1}{p^{\prime}}=1 .
$$

Here $p=\frac{1}{[\alpha]+1-\alpha}$ and $p^{\prime}=\frac{1}{\alpha-[\alpha]}$. Further, using this Hölder inequality and (3.32) we obtain

$$
\begin{aligned}
& \sum_{i<n}(1+i h)^{\gamma-1}\left|v_{j}^{i}\right|_{0}^{2} h \\
& \leq C\left|v_{j}^{0}\right|_{\alpha}^{2([\alpha]+1-\alpha)} \sum_{i<n}(1+i h)^{-([\alpha]+1-\gamma)}\left((1+i h)^{[\alpha]}\left|v_{j}^{i}\right|_{\alpha-[\alpha]-1}^{2}\right)^{\alpha-[\alpha]} h \\
& \leq C\left|v_{j}^{0}\right|_{\alpha}^{2([\alpha]+1-\alpha)}\left[\sum_{i<n}(1+i h)^{\left.\left.-\frac{[\alpha]+1-\gamma}{[\alpha]+1-\alpha}\right]^{[\alpha]+1-\alpha}\left[\sum_{i<n}(1+i h)^{[\alpha]}\left|v_{j}^{i}\right|_{\alpha-[\alpha]-1}^{2}\right)\right]^{\alpha-[\alpha]} h}\right. \\
& \leq C\left|v_{j}^{0}\right|_{\alpha}^{2} h,
\end{aligned}
$$

where $[\alpha]<\gamma<\alpha$ implies $\frac{[\alpha]+1-\gamma}{[\alpha]+1-\alpha}>1$. Thus we have the following from (3.31).

Lemma 3.5. If $\alpha$ is not an integer, then it holds for any $\gamma<\alpha$

$$
(1+n h)^{\gamma}\left\|v_{j}^{n}\right\|^{2}+\sum_{i<n}(1+i h)^{\gamma}\left\|v_{j+1}^{i}-v_{j}^{i}\right\|^{2} \leq C\left|v_{j}^{0}\right|_{\alpha}^{2} .
$$

Combining the latter part of Lemma 3.4 with Lemma 3.5, we complete the proof of Proposition 2.2.

Thus, assuming that $N(0)$ is suitably small, by a standard continuity argument, the problem (2.7) with the initial value $v_{j}^{0}$ has a unique global solution $v_{j}^{n}$ satisfying (2.8) for any $n \geq 0$. Since $N(0)=\left|v_{j}^{0}\right| \leq\left|v_{j}^{0}\right|_{\alpha}$ for $\alpha>0$, Theorem 2.1 follows.

We now turn to prove our main Theorem 1.1.

Proof of Theorem 1.1. First we prove that the condition (1.12) on initial data implies $\left|v_{j}^{0}\right|_{\alpha}$ is small. Here we give a proof under the condition

$$
\sum_{j=-\infty}^{\infty}\left(1+j^{2}\right)^{\kappa}\left|u_{j}^{0}-\phi_{j}\right|^{2} \leq \delta
$$


for any given constant $\kappa>\frac{\alpha}{2}+1$ and $\delta$ is a suitably small constant. Applying the Hölder inequality to

$$
v_{j}^{0}=\sum_{k=-\infty}^{j}\left(u_{k}^{0}-\phi_{k}\right),
$$

we have

$$
\begin{aligned}
\left|v_{j}^{0}\right|^{2} & \leq \sum_{k=-\infty}^{j}\left(1+k^{2}\right)^{\kappa}\left|u_{k}^{0}-\phi_{k}\right|^{2} \sum_{k=-\infty}^{j}\left(1+k^{2}\right)^{-\kappa} \\
& \leq \delta \sum_{k=-\infty}^{j}\left(1+k^{2}\right)^{-\kappa} .
\end{aligned}
$$

Therefore,

$$
\begin{aligned}
\left|v_{j}^{0}\right|_{\alpha}^{2} \leq \sum_{j}\langle j\rangle^{\alpha}\left|v_{j}^{0}\right|^{2} & \leq \delta \sum_{j}\left(1+j^{2}\right)^{\frac{\alpha}{2}} \sum_{k=-\infty}^{j}\left(1+k^{2}\right)^{-\kappa} \\
& \leq \delta \int_{-\infty}^{+\infty}\left(1+x^{2}\right)^{\frac{\alpha}{2}} \int_{-\infty}^{x}\left(1+y^{2}\right)^{-\kappa} d y d x \\
& \leq \frac{\delta}{\kappa-\frac{\alpha}{2}-1} O(1),
\end{aligned}
$$

so

$$
N(0) \leq\left|v_{j}^{0}\right|_{\alpha} \leq O(1) \sqrt{\delta}
$$

Thus the hypothesis in Theorem 2.1 is fulfilled under the condition (1.12). It follows from Theorem 2.1 that there exists a unique global solution, $u_{j}^{n}$, to the L-F scheme (1.1) due to the relation

$$
u_{j}^{n}=\phi_{j}+v_{j}^{n}-v_{j-1}^{n}
$$

which follows from (2.3). Next we study the asymptotic behavior of the solution $u_{j}^{n}$ to (1.1). It follows from (2.7) that

$$
\sum_{n=0}^{\infty}(1+n h)^{\gamma}|| v_{j}^{n}-v_{j+1}^{n} \|^{2}<C\left|v_{j}^{0}\right|_{\alpha}^{2}
$$

which implies

$$
\lim _{n \rightarrow \infty}(1+n h)^{\gamma} \sum_{j}\left|u_{j}^{n}-\phi_{j}\right|^{2}=\lim _{n \rightarrow \infty}(1+n h)^{\gamma} \sum_{j}\left|v_{j}^{n}-v_{j-1}^{n}\right|^{2}=0
$$

and

$$
\left\|v_{j+1}^{n}-v_{j}^{n}\right\| \leq C(1+n h)^{-\frac{\gamma}{2}}\left|v_{j}^{0}\right|_{\alpha} .
$$

Moreover, by virtue of (3.36),

$$
\left|u_{j}^{n}-\phi_{j}\right|=\left|v_{j}^{n}-v_{j-1}^{n}\right| \leq\left\|v_{j+1}^{n}-v_{j}^{n}\right\| .
$$

Combining these facts, we obtain

$$
\sup _{j}\left|u_{j}^{n}-\phi_{j}\right| \leq C(1+n h)^{-\frac{\gamma}{2}}\left|v_{j}^{0}\right|_{\alpha}
$$

which proves Theorem 1.1. 


\section{REFERENCES}

[1] I. L. Chern, Large-time behavior of solutions of Lax-Friedrichs finite difference equations for hyperbolic systems of conservation laws, Math Comp., 56(1991),107-118. MR 91j:65148

[2] M.G. Crandall and A. Majda, Monotone difference approximations for scalar conservation laws, Math. Comp., 34(1980), 1-28. MR 81b:65079

[3] B. Engquist and S. Osher, One-sided difference approximations for nonlinear conservation laws, Math. Comp., 36(1981), 321-351. MR 82c:65056

[4] J. Goodman, Nonlinear asymptotic stability of viscous shock profiles to conservation laws, Arch. Rat. Mech. Anal. 95(1986), 325-344. MR 88b:35127

[5] E Harabetian, Rarefaction and large-time behavior for parabolic equations and monotone schemes, Comm. Math. Phys. 114(1988), 527-536. MR 89d:35084

[6] A.M.Il'in and O.A.Oleinik, Asymptotic behaviour of solutions of the Cauchy problem for certain quasilinear equations for large time, Mat. Sbornik, 51(1960), 191-216. MR 22:11222

[7] G. Jennings, Discrete shocks, Comm.Pure Appl. Math. 27(1974), 25-37. MR 49:3358

[8] C. Jones, R. Gardner and T. Kapitula, Stability of travelling waves for non-convex scalar viscous conservation laws, Comm. Pure Appl. Math., 46(1993)505-526. MR 94c:35123

[9] S. Kawashima and A. Matsumura, Asymptotic stability of travelling wave solutions of system for one-dimensional gas motion, Comm. Math. Phys., 101(1985), 97-127. MR 87h:35035

[10] S. Kawashima and A. Matsumura, Stability of shock profiles in viscoelasticity with non-convex constitutive relations, Comm. Pure Appl. Math. 47(1994), 1547-1569. MR 95h:35136

[11] H.L. Liu, Asymptotic stability of shock profiles for non-convex convection-diffusion equation, (preprint)(1994).

[12] T. P. Liu, Nonlinear stability of shock waves for viscous conservation laws, Mem. Amer. Math. Soc., 56, no.328(1985). MR 87a:35127

[13] H.L. Liu and J.H. Wang, Nonlinear stability of stationary discrete shocks for non-convex scalar conservation laws, Math. Comp. 65(1996), 1137-1153. CMP 95:16

[14] J. G. Liu and Z. P. Xin, Nonlinear stability of discrete shocks for systems of conservation laws, Arch. Rat. Mech. Anal., 125(1993), 217-256. MR 95c:35166

[15] J. G. Liu and Z. P. Xin, $L^{1}$ stability of stationary discrete shocks, Math. Comp. 60(1993), 233-244. MR 93d:35097

[16] A. Majda and J. Ralston, Discrete shock profiles for systems of conservation laws, Comm. Pure Appl. Math. 32(1979), 445-483. MR 81i:35108

[17] A. Matsumura and K. Nishihara, On the stability of travelling wave solutions of a onedimensional model system for compressible viscous gas, Japan. J. Appl. Math., 2(1985), 17-25. MR 87j:35335a

[18] Nishihara,K. A note on the stability of travelling wave solutions of Burgers equation, Japan. J. Appl. Math. 2(1985), 27-35. MR 87j:35335

[19] S. Osher, J. Ralston, $L^{1}$ stability of travelling waves with applications to convective porous medium flow, Comm. Pure Appl. Math. 35(1982), 737-749. MR 84j:35090

[20] D.H. Sattinger, On the stability of waves of nonlinear parabolic systems, Adv. in Math., 22(1976), 312-355. MR 55:8561

[21] R. Sanders, On convergence of monotone finite difference schemes with variable spatial differencing, Math. Comp. 40(1983), 91-106. MR 84a:65075

[22] D. Serre, $L^{1}$ stabilty of viscous shock profile for scalar non-convex conservation law, private communication(1994).

[23] Y. S. Smyrlis, Existence and Stability of stationary profiles of the LW scheme. Comm. Pure Appl. Math., 43(1990), 508-545. MR 91d:65143

[24] A. Szepessy and Z. Xin, Nonlinear stability of viscous shock profiles, Arch. Rat. Mech. Anal. 122(1993) 53-104. MR 93m:35125

[25] E. Tadmor, The large-time behavior of the scalar genuinely nonlinear Lax-Friedrichs scheme, Math. Comp. 43(1984), 353-368. MR 86g:65162

Department of Mathematics, Henan Normal University, XinXiang, 453002, P. R. China E-mail address: guozm@sun.ihep.ac.cn

Institute of Systems Science, Academia Sinica, Beijing 100080, P. R. China

E-mail address: jwang@iss06.iss.ac.cn 